\title{
Commentary on: Outpatient visits versus telephone interviews for postoperative care: a randomized controlled trial
}

\author{
Aiste Ugianskiene ${ }^{1}$ (1) \\ Received: 7 February 2019 / Accepted: 13 March 2019/Published online: 23 March 2019 \\ (C) The International Urogynecological Association 2019
}

This was a randomized controlled trial comparing routine postoperative outpatient clinic visits versus telephone calls for women undergoing surgery for pelvic floor disorders at academic institution in New Mexico from October 2016 to November 2017. The clinic group $(n=50)$ returned for visits 2,6 , and 12 weeks postoperatively, while the telephone group $(n=50)$ received a phone call from a nurse at the same time intervals. Women completed the validated Surgical Consumer Assessment of Healthcare Providers and Systems (S-CAHPS) questionnaire, Pelvic Floor Distress Inventory-20 (PFDI-20), and pain scales prior to the operation and 3 months postoperatively. The authors hypothesized that telephone calls to patients at regular intervals would result in non-inferior satisfaction scores without differences in safety and clinical outcome measures.

The study showed than the S-CAHPS global assessment surgeon rating from patients in the telephone group was noninferior to the clinic group ( $92 \%$ clinic vs. $88 \%$ telephone participants rated their surgeons $9-10$ on S-CAHPS, with a non-inferiority limit of $36.1, p=0.006)$. There was no difference in adverse events between groups $(n=26 ; 57 \%$ from clinic vs. $43 \%$ from telephone group, $p=0.36$ ). Patients in the telephone group did not require additional emergency room or primary care visits compared with the clinic group. Clinical outcome measures improved in both groups during the postoperative period, with no differences between groups (all $p>0.05$ ).

In summary, this study showed that telephone follow-up postoperative care provides high patient satisfaction without sacrificing safety and clinical outcomes. This study highlights the need to consider implementation of innovative care to reduce financial burdens and provide care that is more convenient for our patients.

\section{Compliance with ethical standards}

Conflicts of interest None.

Publisher's note Springer Nature remains neutral with regard to jurisdictional claims in published maps and institutional affiliations.
Aiste Ugianskiene au@rn.dk

1 Aalborg University Hospital, Reberbansgade 15, 9000 Aalborg, Denmark 